\title{
Demystifying Advertising Campaign for CPA Goal Optimization
}

\author{
Deguang Kong, Konstantin Shmakov and Jian Yang \\ Yahoo Research, Oath, 701 1st Ave, Sunnyvale, California, 94086 \\ \{dkong,kshmakov,jianyang\}@oath.com
}

\begin{abstract}
In cost-per-click (CPC) or cost-per-impression (CPM) advertising campaigns, advertisers always run the risk of spending the budget without getting enough conversions. Moreover, the bidding on advertising inventory has few connections with propensity that can reach to cost-per-acquisition (CPA) goals. To address this problem, this paper presents a bid optimization scenario to achieve the desired CPA goals for advertisers. In particular, we build the optimization engine to make a decision by solving the constrained optimization problem. The proposed model can naturally recommend the bid that meets the advertisers' expectations by making inference over history auction behaviors. The bid optimization model outperforms the baseline methods on real-world campaigns, and can be applied into a wide range of scenarios for performance improvement and revenue liftup.
\end{abstract}

\section{CCS CONCEPTS}

- Information systems $\rightarrow$ Computational advertising;

\section{KEYWORDS}

Advertising; Optimization; Bidding

\section{ACM Reference Format:}

Deguang Kong, Konstantin Shmakov and Jian Yang. 2018. Demystifying Advertising Campaign for CPA Goal Optimization. In WWW'18: The 2018 Web Conference Companion, April 23-27, 2018, Lyon, France. ACM, New York, NY, USA, 2 pages. https://doi.org/10.1145/3184558.3186940

\section{INTRODUCTION}

Online advertising uses the internet to deliver the promotional marketing messages provided by advertisers to consumers on the publisher platform by integrating the advertisements into its online content. Bid optimization is a technique to optimize the bidding process, which allows the advertiser to bid for winning the impressions and clicks. The effective and efficient bidding strategies have been proposed to bring more benefits for advertisers, consumes and third-party platforms. For example, for the same key word "buy jeans", there is 20 conversions with $2 \mathrm{CPC}$ bid for advertiser 1 but only 5 conversions with the same price for advertiser 2 . To effectively learn the useful "signals" available for bid adjustment for advertisers, we build effective models to capture what the cost and win rate will look like if the advertisers change the bidding price.

In this paper, we focus on cost per acquisition (CPA) goal, which helps the advertisers programmatically choose the best "smart" bid,

This paper is published under the Creative Commons Attribution 4.0 International (CC BY 4.0) license. Authors reserve their rights to disseminate the work on their personal and corporate Web sites with the appropriate attribution.

WWW' '18, April 23-27, 2018, Lyon, France

() 2018 IW3C2 (International World Wide Web Conference Committee), published under Creative Commons CC BY 4.0 License.

ACM ISBN 978-1-4503-5640-4/18/04.

https://doi.org/10.1145/3184558.3186940 and then derive the most possible scale for the targets, which in turn, acquires more traffic for e-commerce business. We present an effective way to automatically set bids for auctions to get as many conversions as possible within the target CPA goals. Then we can recommend bid to advertisers for adjustment based on how likely a click is to lead to a conversion. It therefore can help advertisers to plan CPA goals and revenue targeting. We believe all the algorithm advancement can bring in more predictive signals and better target accuracy compared to inflection point [4], portfolio optimization [3] and etc. Compared to $\mathrm{CPA}$, cost per mille impressions $(\mathrm{CPM})^{1}$ poses high risk on the conversions, cost per sale (CPS) ignores the value of click which potentially provides worse traffic liquidating, and cost per click (CPC) controls the cost of clicks after running page views without giving enough attention to the true conversions that may actually bring in revenue increases for advertisers.

\section{METHODOLOGY}

An illustrative example. Let's say the advertiser is measuring sales for several online electronic stores by optimizing the bids based on the value of a shopping cart in total. The goal is 50 per conversation (that leads to sales) based on every dollar one spends on ads. If one is setting a target CPA goal as $\$ 50$ - for every $\$ 50$ you spend on ads, you'd like to get 1 conversion (and get the corresponding revenue based on conversion value). More concretely,

Store 1: $\$ 100$ in spend $\div 2$ conversions $=\$ 50 \mathrm{CPA}$ goal

Store $2: \$ 150$ in spend $\div 4$ conversions $=\$ 37.5 \mathrm{CPA}$ goal

Store $3: \$ 500$ in spend $\div 5$ conversions $=\$ 100 \mathrm{CPA}$ goal

Based on these data one would decide to spend more budget at store 1 and store 2, as one is hitting the goal on these websites. At store 3 , the advertiser pays the double of the goal per conversion, so the campaign will stop bidding there.

Optimization Goal A question that naturally follows is: how to set the bid price in order to achieve these CPA goals? More formally, given the CPA goal with value $C$, we aim to provide the bid recommendation that can maximize the clicks while considering CPA goal for the advertiser i, i.e.,

$$
\max _{\text {bid }} C l i c k(\text { bid }) ; \text { s.t } \quad C P A(\text { bid })=C ;
$$

where click(bid) denotes the number of clicks the advertiser can obtain given the bidding price at bid, CPA(bid) tells the CPA goal one can achieve with bidding price $b^{2}{ }^{2}$.

\section{Algorithm Workflow}

\section{(i) Get all supply impressions}

In performance buying scenarios, number of impression one can get from the inventory is determined by a combination of the content of the site and a specific campaign's creative and targeting,

\footnotetext{
${ }^{1}$ As the social media ads, Facebook actually used optimized cost per mille (oCPM) criterion to allow advertisers to bid for click and actually pay per impression

${ }^{2}$ For notation simplicity purpose, advertising campaign $i$ is ignored in the paper next, and all the modeling is applied for the particular campaign $i$.
} 


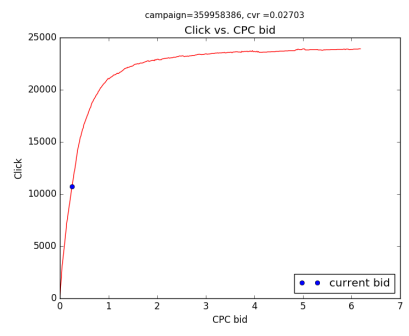

(a) Click vs. bid

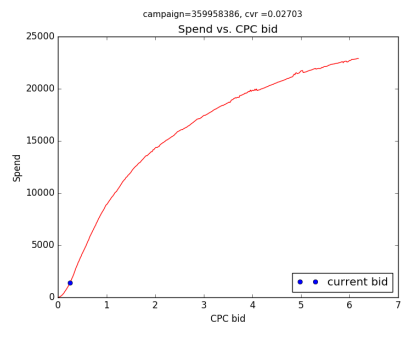

(b) Spend vs. bid

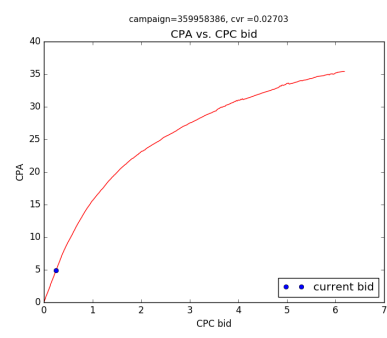

(c) CPA vs. bid

Figure 1: CPA vs. bid, Click vs. bid, Spend vs. bid curves for an advertising campaign. CVR of this ad campaign is 0.0270 .

which is proportional to the available number of impressions on the particular publisher, goe location and targeting attribution settings.

(ii) Click and Conversion prediction

Translating Goals of CPA into click and conversion number prediction is necessary for advertisers, because advertisers typically pay for a particular user response via a cost-per-click (CPC) or CPM. More formally,

$$
\begin{gathered}
\text { Click }(\text { bid })=\text { Impression } \times \text { Winrate }(\text { bid }) \times p C T R, \\
\text { Conversion }(\text { bid })=\text { Click }(\text { bid }) \times p C V R,
\end{gathered}
$$

where Click(bid) tells the number of click the advertiser can obtain at bid price, Impression is the available impressions based on the combination of publisher and targeting information. Winrate(bid) is the winning ratio from all available impressions, Conversion(bid) is the number of conversions obtained at price bid, $p C T R, p C V R$ are the predicted click-through-rate (CTR) [2] and predicted conversionrate (CVR) for the particular advertiser based on history campaigns.

\section{(iii) CPA optimization for bid}

The CPA goal is transformed into CPC bids based on a variety of factors, including historical win rates for a particular type of impression, and actual cost at that bid price. For example, if the historical rates indicate that the win rate is low, then a lower bid will be submitted. On the other hand, if the response rate is high, then a higher bid will be submitted. More formally,

$$
\begin{aligned}
C P A(\text { bid }) & =\frac{\text { Spend }(\text { bid })}{\text { Conversion }(\text { bid })}=\frac{\text { Click }(\text { bid }) \times C P C \_c o s t(\text { bid })}{C l i c k(\text { bid }) \times p C V R} \\
& =\frac{C P C \_ \text {cost }(\text { bid })}{p C V R}=\frac{e C P M_{-} \cos t(\text { bid })}{1000 \times p C T R \times p C V R},
\end{aligned}
$$

where $S$ pend(bid) is the money spent, CPC_cost is the cost-perclick, and eCPM_cost is the cost-per-mille at price bid, respectively.

\section{EXPERIMENT}

Given the fact that we only know the ground-truth for the current bid, i.e., the true number of conversions and the true spend for current campaign at current bid price, we therefore design experiments to compare the differences between the true CPA (denoted as $\mathrm{CPA}$ ) and the forecasted CPA (denoted as $C \hat{P} A$ ) at current bid. More formally, we use the mean absolute percentage error (MAPE) and root mean square percentage error (RMSPE) as the measurement, i.e., $\mathrm{MAPE}=\sum_{i=1}^{n} \frac{\left|y_{i}-\hat{y_{i}}\right|}{y_{i}}, \quad \mathrm{RMSPE}=\sqrt{\frac{1}{n} \sum_{i=1}^{n}\left(\frac{\hat{y_{i}}-y_{i}}{y_{i}}\right)^{2}}$, where $y_{i}$ is the CPA for ads campaign $i$ with current bid $b_{i}$, and $\hat{y}_{i}$ is the forecasted CPA for campaign $i$ at current bid $b_{i}$. The smaller values of MAPE, RMSPE indicate better performance. We also compare the baselines: (a) Nearest Neighbor Search (NNS). If the observed
Table 1: Performance comparisons of MAPE, RMSPE between CPA and predicted $C \hat{P} A$ using (1) proposed method; (2) baseline 1: nearest neighbor search (NNS); (3) baseline 2: linear interpolation $(\mathrm{LI})$

\begin{tabular}{c|c|c}
\hline \hline Method & MAPE & RMSPE \\
\hline Our method & $10.28 \%$ & $15.68 \%$ \\
Nearest Neighbor Search (NNS) & $23.86 \%$ & $50.23 \%$ \\
Linear Interpolation (LI) & $18.65 \%$ & $32.65 \%$ \\
\hline \hline
\end{tabular}

bid price exists in history, then we retrieve the corresponding CPA computed using spend and conversion in history. Otherwise, we use the nearest neighbor method to predict the CPA. For the observed bid $b$, if $b_{i}^{\prime}$ is the nearest neighbor of bid $b_{i}$ in history, then we retrieve the corresponding CPA for bid $b_{i}^{\prime}$ as the predicted CPA; (b) Linear interpolation (LI) The idea is to use linear interpolation in historical bid price to predict CPA correspondingly ${ }^{3}$. Table. 1 shows the performance evaluation on observed 190,876 ad campaigns. Given that it is a challenging task to predict CPA for diversified, fluctuated ad campaigns, the result obtained using proposed model is competitive and reasonable, because it leverages all history information completely by smartly setting the bid price. Fig. 1 shows the example of ad campaigns with forecasted CPA, total money of spend and number of clicks at different bid prices.

\section{CONCLUSION}

This paper presents a novel way for bid forecasting to achieve the desired CPA goals, which we believe is an important piece that powers the bid landscape for bid recommendation and forecasting. The approach is interesting in providing the optimal bid solution based on targeting CPA goals using history performance data. We also provide effective optimization strategy with demonstrated performance improvement.

\section{REFERENCES}

[1] Ying Cui, Ruofei Zhang, Wei Li, and Jianchang Mao. 2011. Bid landscape forecasting in online ad exchange marketplace. In SIGKDD'11. 265-273.

[2] Hongchang Gao, Deguang Kong, Miao Lu, Xiao Bai, and Jian Yang. 2018. ContextAware Attention Convolutional Neural Network for Advertiser-Level CTR Forecasting. In WWW'2018. ACM, to appear.

[3] Deguang Kong, Xiannian Fan, Konstantin Shmakov, and Jian Yang. 2018. A combinational optimization approach for advertising budget allocation. In WWW'2018. ACM, to appear.

[4] Deguang Kong, Konstantin Shmakov, and Jian Yang. 2018. Demystifying Advertising Campaign for CPA Goal Optimization. In WWW'2018. ACM, to appear.

${ }^{3}$ Note that a regression model (e.g., Gradient Boost Decision Tree method (GBDT) ) for CPA prediction can be built using the features including the bid price, advertiser ID and other advertiser features (such as supply group, advertiser category, etc) [1]. The detailed comparisons will be added in full paper. 